\title{
Biasing Enantiomorph Formation via Geometric Confinement: Nanocorrals for Chiral Induction at the Liquid-Solid Interface
}

\author{
Johannes Seibel, Lander Verstraete, Brandon E. Hirsch, Ana M. Bragança and Steven De Feyter* \\ Department of Chemistry, Division of Molecular Imaging and Photonics, KU Leuven, Celestijnenlaan 200F, B-3001 \\ Leuven, Belgium
}

Scanning Tunneling Microscopy, Chirality, Self-assembly, Covalent Surface Modification

Supporting Information Placeholder

\begin{abstract}
Nanocorrals created by scanning probe lithography on covalently modified graphite surfaces are used to induce a chiral bias in the enantiomorphic assembly of a prochiral molecule at the liquid/graphite interface. By controlling the orientation of the nanocorrals with respect to the underlying graphite surface, the nanocorral handedness can be freely chosen and thus a chiral bias in molecular self-assembly is created at an achiral surface solely by the scanning probe lithography process.
\end{abstract}

Chiral surfaces play an important role as substrates in chiral separation $^{1}$ and enantioselective heterogeneous catalysis ${ }^{2,3}$ and are most often created through physisorption of either chiral or prochiral molecules. 4 The self-assembly of these molecules into ordered monolayers can then be characterized with sub-molecular resolution using scanning tunneling microscopy (STM). 5 Prochiral molecules often assemble on a surface in mirror domains, and thus the surface remains "globally" achiral. ${ }^{6}$ Globally chiral surfaces formed by prochiral molecules can be created or by the co-adsorption of enantiopure molecules via a sergeants-and-soldiers ${ }^{7,8}$ mechanism or via chiral solvents ${ }^{9-12}$. Beyond the molecular system, (surface) confinement and boundary conditions may impact the induction of chirality too. For example, chiral domain boundaries on metallic substrates resulting from kinks in step edges have been shown to steer the chirality of adjacent domains formed by a prochiral molecule, ${ }^{13}$ as well as the enantioselective adsorption of amino acids. ${ }^{14}$ Further, the enantiospecific adsorption of amino acids on naturally chiral metal surfaces has been studied intensively. ${ }^{15,16}$

Geometric confinement is known to alter the properties and structures of polymers and is an important instrument to influence liquid crystal (LC) structures, ${ }^{17}$ i.e. it has been shown to induce spontaneous symmetry breaking. As a result, achiral nematic LCs can form chiral structures when confined in cylindrical capillaries ${ }^{18,19}$ or tactoidal droplets. ${ }^{20}$ In these cases, however, there was no control over the handedness resulting from the symmetry breaking.

Here, we show biased enantiomorphous monolayer formation of a prochiral molecule (5-octadecyloxy-isophthalic acid, ISA-OC $\mathrm{C}_{18}$ ) via geometric confinement in nanocorrals at the achiral interface between a liquid (octanoic acid, OA) and covalently modified highly oriented pyrolytic graphite (CMHOPG, Fig. 1). The nanocorrals of well-defined size, shape and orientation are fabricated through an STM nanoshaving process locally removing the covalently bound molecules.
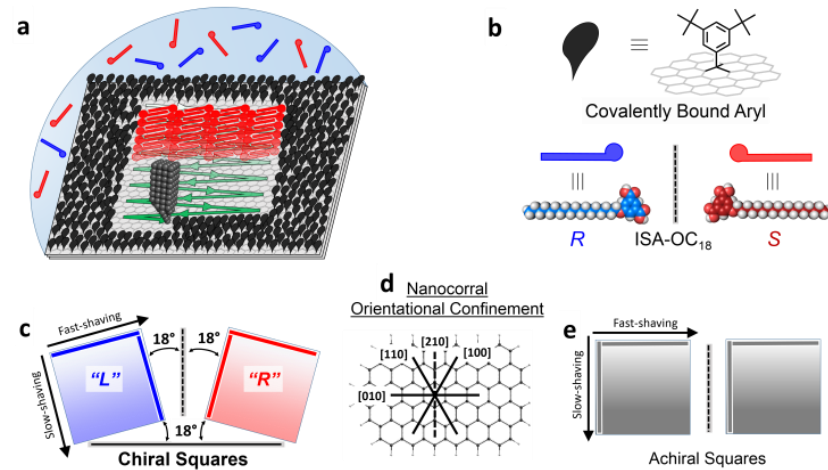

Figure 1. Schematic for the in-situ nanoshaving procedure and the creation of orientationally controlled nanocorrals. Covalently bound aryls are removed by the STM tip in the presence of a ISA$\mathrm{OC}_{18} / \mathrm{OA}$ solution (a). When prochiral ISA-OC 18 molecules adsorb, they obtain either $R$ (blue) or $S$ (red) chirality (b) as the HOPG surface is gradually exposed by nanoshaving. Controlling the orientation of the corrals with respect to the major symmetry axes of graphite (d) allows the generation of enantiomeric pairs of chiral squares, named " $L$ " (blue) and " $R$ " (red), respectively (c). Achiral squares are aligned along major axes of graphite (e). Nanoshaving occurs during the forward movement in raster scanning fashion (line by line). "Fast-shaving" and "slow-shaving" refer to the inline motion $(400 \mathrm{~nm} / \mathrm{s})$ and line-to-line motion $(2 \mathrm{~nm} / \mathrm{s})$ of the STM tip, respectively.

Chiral character is imparted to the nanocorrals through the borders of the corral and the raster nanoshaving process, both of which define oblique angles with respect to high-symmetry surface lattice directions of the underlying HOPG (Fig. 1c-e). By changing the orientation of the confinement container relative to the substrate symmetry, square nanocorrals with opposite tilt angles become an enantiomeric pair, denoted " $L$ " and " $R$ ", which rotate 18 o left and right, respectively, from the [210] surface direction (Fig. 1c). They are therefore coined "chiral squares". Achiral square nanocorrals have boundaries parallel to surface symmetry directions. The fast-shaving direction of the raster scan is parallel to the top-boundary of the 
nanocorral, while the slow-shaving direction defines the side boundaries.

The self-assembly of ISA-OC ${ }_{18}$ molecules into chiral lamellae with interdigitated alkyl-chains has been studied previously. ${ }^{21,22}$ Prochiral ISA-OC ${ }_{18}$ molecules can either adsorb with $R$ or $S$ chirality (Fig. 1b). Slip-stacking of molecules with $S(R)$ chirality generates lamellae that tilt to the right (left), respectively (Fig. 2a).
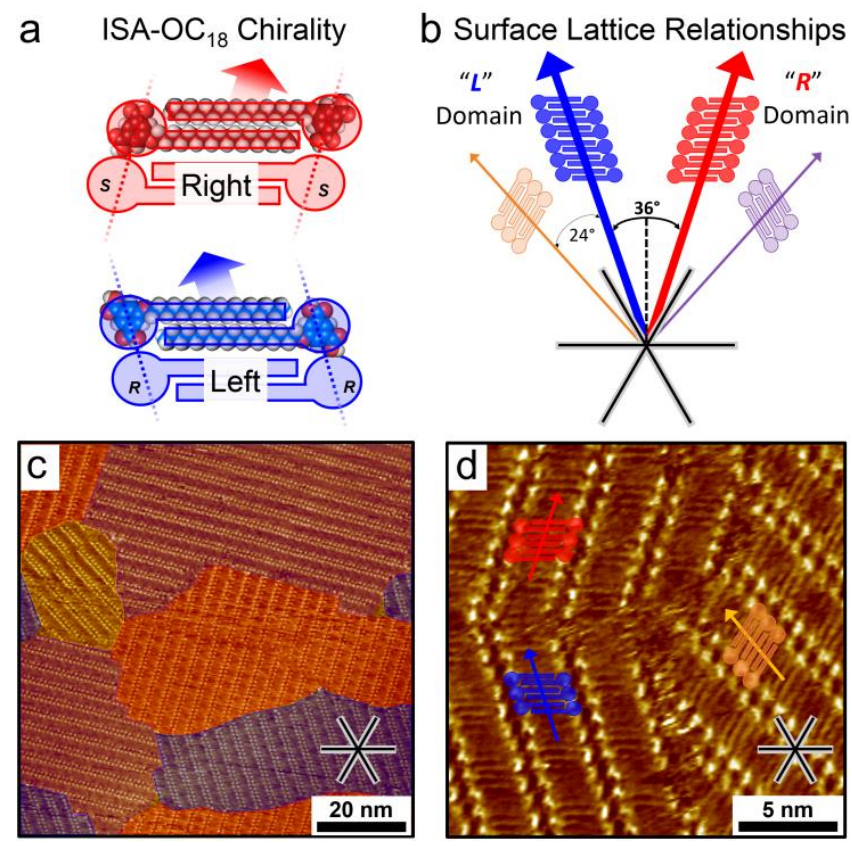

Figure 2. Schematic illustrating the chiral self-assembly of ISA$\mathrm{OC}_{18}$ and representative STM images at the OA/HOPG interface. a) Molecules with $S$ chirality (red) slip-stack to form lamellae that tilt to the right, while molecules with $R$ chirality (blue) form left tilted lamellae. b) Schematic outlining the lamellae orientation with respect to the HOPG surface lattice directions. The domain with lamellae tilted left with respect to [210] surface direction (dotted line) is denoted " $L$ " domain and the right tilted lamellae " $R$ " domain, respectively, which include an angle of $36^{\circ}$. Rotational " $R$ " and " $L$ " domains are marked orange and violet. c) Large-area STM image showing mirror and rotational domains. The enantiomorphs are marked in red, blue, orange, and violet respectively, corresponding to the colors in b. d) Small-scale STM image showing left and right "mirror" domains (red and blue) and one rotational domain (orange). Imaging conditions: $V_{b}=-0.7 \mathrm{~V}, I_{t}=70 \mathrm{pA}$

Although the alkyl-chains in both of these mirror domains are oriented along the same major symmetry axis of HOPG, the angle between the enantiomorphous lamellae mirrored at the normal of a main symmetry direction (e.g. [210]) is $36^{\circ}$ (Fig. 2b). STM overview and zoom-in images highlight these organizational packing differences observed for ISA-OC ${ }_{18}$ (Fig. $2 \mathrm{c}$ and $2 \mathrm{~d}$ ). In our experiments, we target chiral selection between left and right domains mirrored at the surface normal. It is important to note, however, that the angle between enantiomorphs mirrored at a high-symmetry direction (e.g. [10o]) measures only $24^{\circ}$. Such a symmetry operation also involves molecular rotation and hence these domains are referred to as rotational domains.

Nanoconfinement experiments are carried out on CMHOPG. The HOPG surface is densely modified with chemisorbed 3,5-bis-tert-butylphenyl molecular units (Fig. 1) using electrochemical grafting of diazonium salts. ${ }^{23}$ After the covalent modification, a droplet of $0.2 \mathrm{mM}$ solution of ISA-OC ${ }_{18}$ in OA is drop-casted onto the CM-HOPG. When nanoshaving is performed in situ, i.e. in the presence of ISA-OC $\mathrm{C}_{18}$ solution, the restored pristine HOPG gradually becomes available for molecular self-assembly (Fig. S1). Confinement experiments consist of arrays of four nanocorrals, where the nanoshaving procedure is done first with tunneling conditions that bring the tip close to the surface, i.e. voltage bias $\left(V_{b}\right)$ of $-0.001 \mathrm{~V}$ and tunneling current $\left(I_{t}\right)$ of $200 \mathrm{pA}$. STM imaging after nanoshaving is done with relatively mild tunneling conditions, $\left(V_{b}\right)$ of $-0.7 \mathrm{~V}$ and $\left(I_{t}\right)$ of 50-70 pA. No differences were observed in the $2 \mathrm{D}$ packing structure of ISA-OC $\mathrm{C}_{18}$ inside the nanocorrals (Fig. 3) compared to bare HOPG (Fig. S2). Only nanocorrals with a single domain were taken into account. Even though some of the nanocorrals showed deviations from the ideal shape (yellow arrows in Fig. 3), we did not exclude any corrals from the statistics to avoid any unintended selection bias. Note that experiments were performed with four squares of the same orientation in one image to reduce the effect of drift (Fig. S3).

Representative STM images of " $L$ " and " $R$ " handed nanocorrals are shown in Fig. $3 a$ and $3 b$. Statistical analysis of more than 500 nanocorrals over the course of 11 experimental sessions revealed enantiomorph selection of left-handed domains with $65 \%$ probability for square nanocorrals with " $L$ " chirality (Fig. 3a). The ISA-OC ${ }_{18}$ lamellae of left-handed domains containing $R$ molecules are parallel to the slow-shaving boundary of " $L$ "-handed nanocorrals. Right-handed domains, however, still make up a fair portion of the assembly with $\sim 27 \%$. Other rotational domains are observed in much smaller proportions (Fig 3a, orange and black). On the other hand, nanocorrals with " $R$ "-handedness predominately contain right-handed domains (58\%, Fig. 3b). Although subtle, a clear chiral bias is introduced between the two enantiomorphs by the nanocorral surface orientation.

Control experiments were then conducted with achiral squares. The statistical analysis of 171 achiral squares showed no clear chiral biasing effect (Fig. 3c). For these achiral squares, the fast-shaving direction of the nanocorral is aligned parallel to the alkyl-chains of ISA-OC ${ }_{18}$. As a result, the number of rotational domains with alkyl-chains not aligned with the fast-shaving direction is virtually eliminated (Fig. 3c, black bar), indicating the importance of the long molecular axis for preferential domain orientation. ${ }^{24,25}$

Alternative opportunities for chiral selection are parallelogram-shaped nanocorrals with either chiral top or side boundaries. However, we did not observe any clear bias in nanocorrals with only chiral side- or top boundaries (Fig. S4-S6). Additionally, we performed experiments to examine the influence of nanocorral size. In smaller squares a bias towards the same enantiomorphs was observed (Fig. S7). However, an increase in rotational domains and thus smaller bias was observed, which is in agreement with previous results. ${ }^{24}$ In larger squares the chiral biasing effect seems to be lost (Fig. S8). 


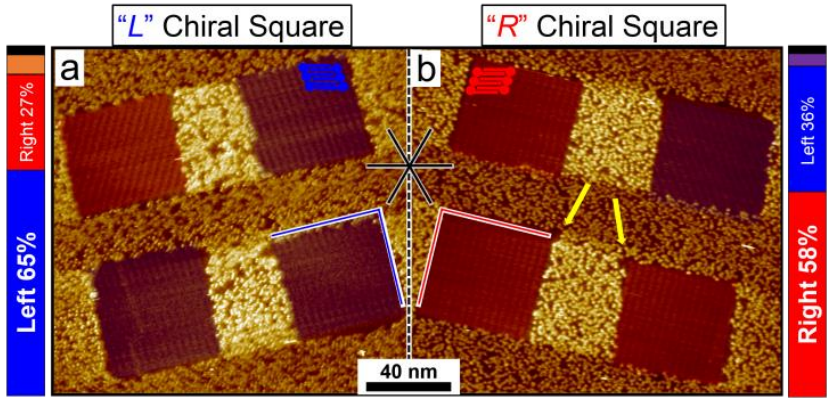

Achiral Square

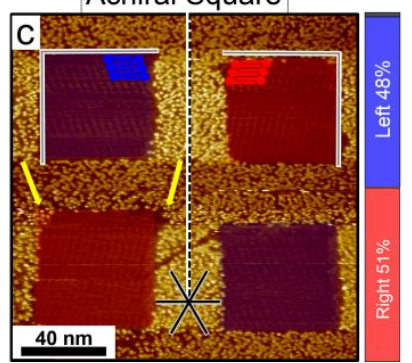

Figure 3. Representative STM images of chiral square nanocorrals with " $L$ " (a) and " $R$ " (b) handedness as well as achiral squares (c). For the chiral squares a preferred formation of the enantiomorph aligned parallel to the slow-shaving direction is observed (see bargraphs next to the STM images). The second most frequent domain is the mirror domain, followed by the rotational domain with the lowest angle with respect to the square borders (orange in a, purple in b). Other rotational domains are rarely observed and summarized in the black bar. In the achiral squares the " $L$ " and " $R$ " enantiomorphs are found with equal probability while the amount of rotational domains is further decreased (c). The yellow arrows point at the occasional imperfections at nanocorral borders. Imaging conditions: $V_{b}=-0.7 \mathrm{~V}, I_{t}=60 \mathrm{pA}$

The session-to-session observed variance in the domain distribution (Fig. S6 and S9) highlights the fragility of the chiral biasing processes and indicates that the induction mechanism is subject to experimental variations. That is, the nanocorral borders are not perfectly straight (Fig. S1o) and defects and molecular imperfections can be found at the corral borders (e.g. Fig. 3b). Furthermore, it has been shown that STM tips can be intrinsically chiral, which potentially could also impact a chiral bias. ${ }^{26}$ Notwithstanding these factors, it is clearly proven that chiral induction takes place in chiral squares.

Nanomechanical symmetry breaking should affect both enantiomorphs equally and thus the nanoshaving process itself should not directly impact the way a prochiral molecule adsorbs on a surface. ${ }^{27}$ Although it has been shown that interactions between tecton monomers and confinement borders can be strong enough to guide their orientations, ${ }^{28}$ the interactions between ISA-OC $\mathrm{C}_{18}$ and grafted molecules at the corral borders are expected to be low ${ }^{22}$ and thus should not guide the assembly process. Rather, confinement conditions during corral formation are believed to manipulate kinetic and thermodynamic factors. From a thermodynamic standpoint, molecular packing inside corrals with matching chiral borders is preferred over corrals with conflicting chirality. Idealized schematics of corrals measuring $50 \mathrm{~nm}$ show that the assembly of more molecules is destabilized or disrupted by the chiral mismatched borders compared to corrals with chiral matching border (Fig. 4). Another notable difference is the number of lamellae connecting to the top corral border, i.e. 15 in chiral matching and only 13 in mismatching corrals.

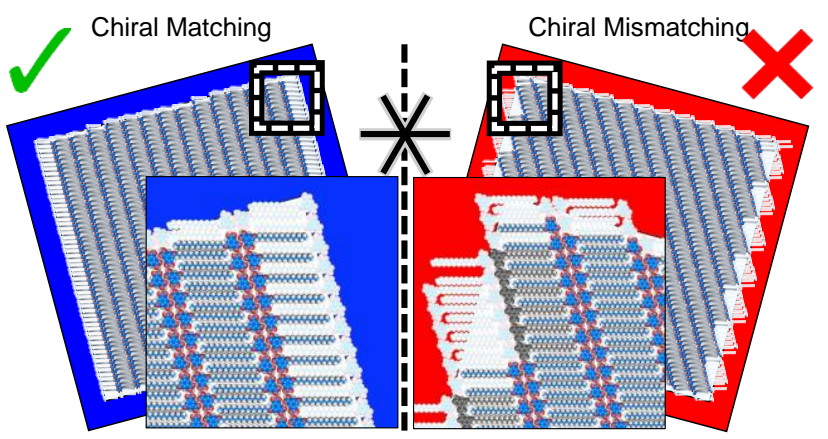

Figure 4. Schematics of the molecular assembly of the " $L$ " ISA$\mathrm{OC}_{18}$ enantiomorph in chiral matching (left) and mismatching (right) nanocorrals. In the chiral mismatching corrals the assembly of roughly $15 \%$ of the molecules is perturbed, i.e. the molecules do not fit in the corral or do not have neighboring molecules to form hydrogen bonds, compared to only $10 \%$ in the chiral matching corrals (faded molecules).

Molecular assembly, however, is thought to occur as the corral is being formed during the nanoshaving process, i.e. after a stable nucleus has formed, only molecules with matching handedness successively attach to the domain. This hypothesis is supported by multiple domain corrals, where the domains typically touch the top boundary while the chiral bias is lost (Fig. S11 and S12). Thus, kinetic effects that impact seed formation and growth are also critically influencing the observed chiral bias. The initial stages of corral formation are considered to alter the seed stability between the two relevant enantiomorphs (Fig. S13). An assembly seed that matches the handedness of the corral experiences shape and orientational matching within the confinement container and should have a higher stability. Alternatively, seeds inside nanocorrals with conflicting handedness experience more destabilizing perturbations at the borders of the initial corral and during growth new lamellae have to form while others collide with the corral border. Since the stability of nucleation seeds is likely to depend on their size, ${ }^{29}$ we propose that nuclei formed in chiral matching nanocorrals are more stable. Chiral conflicting seeds are less stable inside the corral and more likely to dissolve and allow formation of seeds matching the corral handedness. In contrast, the nanoshaving process for achiral squares creates equal situations for both enantiomorphic possibilities (Fig. S14).

In conclusion, we have presented a new method to create chiral structures at an achiral surface to induce a chiral bias in the self-assembly of prochiral molecules. The chirality creation is purely based on the nanoshaving-induced nanocorral shape and orientation with respect to the underlying surface. The possibility to freely choose the nanocorral shape and orientation with respect to the surface and thus bias enantiomorph formation makes this method a valuable tool to study chiral self-assembly phenomena on surfaces. This can for instance be extended to the enantioselective adsorption from a racemic mixture. Furthermore, changing the nature of the grafted molecules, i.e. using chiral ones, will also broaden the versatility of this new approach.

\section{ASSOCIATED CONTENT}

\section{Supporting Information}


The Supporting Information is available free of charge on the ACS Publications website: Experimental methods, supporting figures, and statistics

\section{AUTHOR INFORMATION}

\section{Corresponding Author}

*steven.defeyter@kuleuven.be

\section{Notes}

The authors declare no competing financial interests.

\section{ACKNOWLEDGMENT}

This work has received funding from the Fund of Scientific Research-Flanders (FWO), KU Leuven, and ERC Grant Agreement No. 340324. J.S. acknowledges a Marie Skłodowska-Curie Individual Fellowship (EU project 789865 - EnSurf). LV. and B.E.H thank the FWO for their personal fellowship. B.E.H. also acknowledges the Belgian American Educational Foundation.

\section{REFERENCES}

(1) Lorenz, H.; Seidel-Morgenstern, A. Angew. Chem. Int. Ed. 2014, 53, 1218.

(2) Mallat, T.; Orglmeister, E.; Baiker, A. Chem. Rev. 2007, 107, 4863.

(3) Perez-Garcia, L.; Amabilino, D. B. Chem. Soc. Rev. 2007, 36, 941

(4) Zaera, F. Chem. Soc. Rev. 2017, 46, 7374.

(5) Elemans, J. A.; Lei, S.; De Feyter, S. Angew. Chem. Int. Ed. $2009,48,7298$.

(6) Raval, R. Chem. Soc. Rev. 2009, 38, 707

(7) Parschau, M.; Romer, S.; Ernst, K.-H. J. Am. Chem. Soc. 2004 126, 15398.

(8) Tahara, K.; Yamaga, H.; Ghijsens, E.; Inukai, K.; Adisoejoso, J.; Blunt, M. O.; De Feyter, S.; Tobe, Y. Nat. Chem. 2011, 3, 714.

(9) Destoop, I.; Ghijsens, E.; Katayama, K.; Tahara, K.; Mali, K. S.; Tobe, Y.; De Feyter, S. J. Am. Chem. Soc. 2012, 134, 19568.
(10) Katsonis, N.; Xu, H.; Haak, R. M.; Kudernac, T.; Tomovic, Z.; George, S.; Van der Auweraer, M.; Schenning, A. P.; Meijer, E. W.; Feringa, B. L.; De Feyter, S. Angew. Chem. Int. Ed. 2008, 47, 4997.

(11) Destoop, I.; Minoia, A.; Ivasenko, O.; Noguchi, A.; Tahara, K.; Tobe, Y.; Lazzaroni, R.; De Feyter, S. Faraday Discuss. 2017, 204, 215.

(12) Chen, T.; Li, S.-Y.; Wang, D.; Wan, L.-J. Sci. Adv. 2017, 3, e1701208.

(13) Teugels, L. G.; Avila-Bront, L. G.; Sibener, S. J. J. Phys. Chem. C 2011, 115, 2826.

(14) Kuhnle, A.; Linderoth, T. R.; Besenbacher, F. J. Am. Chem. Soc. 2005, 128, 1076

(15) Gellman, A. J.; Huang, Y.; Koritnik, A. J.; Horvath, J. D. J. Phys. Condens. Matter. 2017, 29, 034001.

(16) Horvath, J. D.; Gellman, A. J. J. Am. Chem. Soc. 2002, 124, 2384.

(17) Ryu, S. H.; Yoon, D. K. Liquid Crystals 2016, 43, 1951.

(18) Nayani, K.; Chang, R.; Fu, J.; Ellis, P. W.; Fernandez-Nieves, A.; Park, J. O.; Srinivasarao, M. Nature Commun. 2015, 6, 8067.

(19) Dietrich, C. F.; Rudquist, P.; Lorenz, K.; Giesselmann, F. Langmuir 2017, 33, 5852.

(20) Tortoraa, L.; Lavrentovicha, O. D. Proc. Natl. Acad. Sci. 2011, 108, 5163.

(21) Tao, F.; Bernasek, S. L. Surf. Sci. 2007, 601, 2284.

(22) Bragança, A. M.; Greenwood, J.; Ivasenko, O.; Phan, T. H.; Mullen, K.; De Feyter, S. Chem. Sci. 2016, 7, 7028.

(23) Greenwood, J.; Phan, T. H.; Fujita, Y.; Li, Z.; Ivasenko, O.; Vanderlinden, W.; Gorp, H. V.; Frederickx, W.; Lu, G.; Tahara, K.; Tobe, Y.; Uji-i, H.; Mertens, S. F. L.; De Feyter, S. ACS Nano 2015, 9,5520 .

(24) Verstraete, L.; Greenwood, J.; Hirsch, B. E.; De Feyter, S. ACS Nano 2016, 10, 10706.

(25) Takajo, D.; Nemoto, T.; Kurata, H.; Isoda, S.; Ozaki, H.; Mazaki, Y. Thin Solid Films 2003, 438, 428.

(26) Tierney, H. L.; Murphy, C. J.; Sykes, E. C. Phys. Rev. Lett. 2011, 106, 010801 .

(27) Hong, L.; Nishihara, T.; Hijikata, Y.; Miyauchi, Y.; Itami, K. Sci. Rep. 2018, 8, 2333.

(28) Fan, Q.; Dai, J.; Wang, T.; Kuttner, J.; Hilt, G.; Gottfried, J. M.; Zhu, J. ACS Nano 2016, 10, 3747.

(29) Seibel, J.; Parschau, M.; Ernst, K. H. J. Am. Chem. Soc. 2015, 137,7970 . 


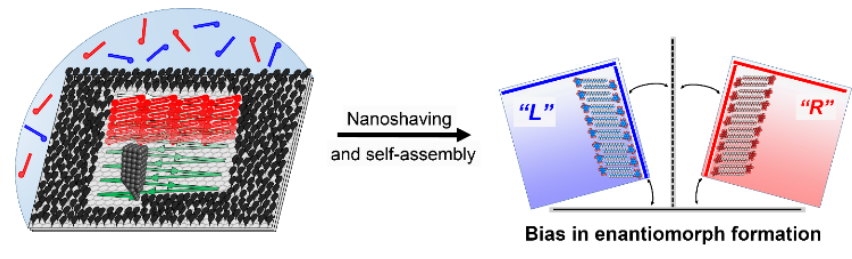

